\title{
BMJ Open \\ The predictive value of serial changes in diaphragm function during the spontaneous breathing trial for weaning outcome: a study protocol
}

\author{
Pengmin Zhou, ${ }^{1}$ Zhongheng Zhang, ${ }^{1}$ Yucai Hong, ${ }^{1}$ Huabo Cai, ${ }^{1}$ Hui Zhao, ${ }^{1}$ \\ Peifeng Xu, ${ }^{2}$ Yiming Zhao, ${ }^{1}$ Shengping Lin, ${ }^{1}$ Xuchang Qin, ${ }^{1}$ JiaWei Guo, ${ }^{1}$ Yun Pan, ${ }^{1}$ \\ Junru Dai ${ }^{1}$
}

To cite: Zhou P, Zhang Z, Hong Y, et al. The predictive value of serial changes in diaphragm function during the spontaneous breathing trial for weaning outcome: a study protocol. BMJ Open 2017;7:e015043. doi:10.1136/ bmjopen-2016-015043

- Prepublication history for this paper is available online. To view these files please visit the journal online (http://dx.doi. org/10.1136/bmjopen-2016015043)

Received 10 November 2016 Revised 17 April 2017 Accepted 23 May 2017

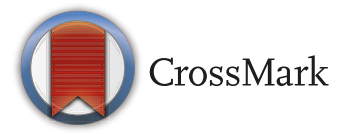

${ }^{1}$ Department of Emergency Medicine, Sir Run Run Shaw Hospital, Zhejiang University School of Medicine, Hangzhou, China

${ }^{2}$ Department of Respiratory Therapy Medicine, Sir Run Run Shaw Hospital, Zhejiang University School of Medicine, Hangzhou, China

Correspondence to

Yucai Hong; zrhyc@hotmail.com

\section{ABSTRACT}

Introduction There is a variety of tools being used in clinical practice for the prediction of weaning success from mechanical ventilation. However, their diagnostic performances are less than satisfactory. The purpose of this study is to investigate the value of serial changes in diaphragm function measured by ultrasound during the spontaneous breathing trial (SBT) as a weaning predictor.

Methods and analysis This is a prospective observational study conducted in a 10-bed medical emergency intensive care unit (EICU) in a universityaffiliated hospital. The study will be performed from November 2016 to December 2017. All patients in the EICU who are expected to have mechanical ventilation for more than 48 hours through endotracheal tube are potentially eligible for this study. Patients will be included if they fulfil the criteria for SBT. All enrolled patients will be ventilated with an Evita-4 by using volume assist control mode prior to SBT. Positive end-expiratory pressure (PEEP) will be set to $5 \mathrm{cmH}_{2} \mathrm{O}$ and fractional inspired oxygen $\left(\mathrm{FiO}_{2}\right)$ will be set to a value below 0.5 that guarantees oxygen saturation by pulse oximetry $\left(\mathrm{SpO}_{2}\right)$ greater than $90 \%$. Enrolled patients will undergo SBT for 2 hours in semirecumbent position. During the SBT, the patients will breathe through the ventilator circuit by using flow triggering $(2 \mathrm{~L} / \mathrm{min})$ with automatic tube compensation of $100 \%$ and $5 \mathrm{cmH}_{2} \mathrm{O}$ PEEP. The $\mathrm{FiO}_{2}$ will be set to the same value as used before SBT. If the patients fail to tolerate the SBT, the trial will be discontinued immediately and the ventilation mode will be switched to that used before the trial. Patients who pass the 2-hour SBT will be extubated. Right diaphragm excursion and bilateral diaphragm thickening fraction will be measured by ultrasonography during spontaneous breathing. Images will be obtained immediately prior to the SBT, and at 5, 30, 60, 90 and 120 min after the initiation of SBT. Rapid shallow breathing index will be simultaneously calculated at the bedside by a respiratory nurse.

Ethics and dissemination The study protocol is approved by the ethics committee of Sir Run Run Shaw Hospital, an affiliate of Zhejiang University, Medical College. The results will be published in a peer-reviewed journal and shared with the worldwide medical community.

Trial registration number ISRCTN42917473; Pre-results.
Strengths and limitations of this study

- This is the first study to investigate the serial changes in diaphragm function during the spontaneous breathing trial. It is possible to find a better predictor for weaning outcome.

- Bedside ultrasound is used to assess the function of the diaphragm in this study, which is simple, rapid and non-invasive.

- The study is limited by the fact that we do not compare the ultrasound result with transdiaphragmatic pressure, and the latter is considered as the gold standard of diaphragm function.

\section{INTRODUCTION}

Approximately $20 \%$ of mechanically ventilated patients have difficulties in weaning from mechanical ventilation, ${ }^{1}$ and about $40 \%$ of ventilation time is spent on the mechanical ventilation weaning process. ${ }^{2}$ Respiratory parameters such as breathing frequency, minute ventilation, maximum inspiratory pressure, tracheal airway occlusion pressure $0.1 \mathrm{~s}$ and a combined index named CROP (compliance, rate, $\mathrm{O}_{2}$, pressure index) have been employed to determine the optimal timing of weaning from mechanical ventilation. ${ }^{3}$ Yang and Tobin ${ }^{4}$ found that the most accurate predictor of weaning outcome was rapid shallow breathing index (RSBI). However, the threshold of RSBI varied in different studies, ${ }^{5-7}$ owing to the differences in ventilate settings, age, gender, body position, underlying illness and endotracheal size. ${ }^{8-10}$ It was reported that RSBI measured early during a spontaneous breathing trial (SBT) is inaccurate in predicting weaning success in patients with chronic obstructive pulmonary diseases. ${ }^{11}$ Also RSBI is not a good predictor for patients whose primary problem is related to poor cough, increased secretion 
and compromised airway protection. These conditions are commonly seen in neurosurgical patients. ${ }^{12} 13$

Recently, some authors have proposed that ultrasonography might be useful during weaning from mechanical ventilation. ${ }^{14}$ Excursion and thickening fraction of the diaphragm has been measured by bedside ultrasonography and was found to be able to quantitatively evaluate diaphragm function, which was useful in predicting weaning outcome. ${ }^{15-19}$ However, these indices are usually measured at the start of SBT. To the best of our knowledge, there is no report on the impact of serial changes in diaphragm excursion and thickening fraction during the SBT on weaning outcomes. The purpose of this study is to investigate whether serial changes in diaphragm function measured by ultrasonography during the SBT could be used to predict the weaning outcome.

\section{METHODS AND ANALYSIS \\ Study design}

This is a prospective observational study conducted in a 10-bed medical intensive care unit (ICU) in a hospital with about 2400 beds. The hospital is an academic teaching hospital affiliated to Zhejiang University. Our emergency intensive care unit enrolled approximately 600 patients who are critically ill annually, and patients with invasive mechanical ventilation accounted for $40 \%$ of the total admissions. The study will start from November 2016, and is planned to end in December 2017. All patients will be consecutively enrolled and followed up for the entire hospital stay.

\section{Study population}

All patients in the ICU who are mechanically ventilated for more than 48 hours through endotracheal tube are potentially eligible for this study. Patients will be included if they meet all of the following criteria: (1) adequate cough, absence of excessive tracheobronchial secretion, resolution of underlying critical illness for which the patient is intubated; (2) the patient is alert and cooperative, without sedation; (3) haemodynamically stable (ie, heart rate $\leq 140 \mathrm{bpm}$, systolic blood pressure between 90 and $160 \mathrm{~mm} \mathrm{Hg}$, without vasopressors); (4) stable metabolic status; and (5) improved respiratory function: arterial oxygen saturation $\left(\mathrm{SaO}_{2}\right)>90 \%$ on fractional inspired oxygen $\left(\mathrm{FiO}_{2}\right) \leq 0.4$ (or oxygen index $\left(\mathrm{PaO}_{2} /\right.$ $\left.\mathrm{FiO}_{2}\right) \geq 150 \mathrm{~mm} \mathrm{Hg}$ ), positive end-expiratory pressure (PEEP) $\leq 8 \quad \mathrm{cmH}_{2} \mathrm{O}$, respiratory rate $\leq 35 \mathrm{bpm}$, without respiratory acidosis. ${ }^{1}$ After enrolment, the patients will undergo an SBT. Subjects will be excluded if they have a history of diaphragm paralysis, cervical spine injury, neuromuscular diseases, a current thoracostomy, pneumothorax or pneumomediastinum.

\section{Study protocol and some definitions}

Patients who meet the above-mentioned criteria will be followed up. Demographics such as sex, age on admission, body mass index (BMI), underlying diseases, reasons for tracheal intubation, Acute Physiology and Chronic Health
Evaluation II (APACHE II) and duration of mechanical ventilation will be recorded. ECG, heart rate, arterial blood pressure and oxygen saturation by pulse oximetry $\left(\mathrm{SpO}_{2}\right)$ will be continuously monitored. All enrolled patients will be ventilated with an Evita-4 (Draeger, Lubeck, Germany) by using volume assist control mode prior to SBT. PEEP will be set to $5 \mathrm{cmH}_{2} \mathrm{O}$ and $\mathrm{FiO}_{2}$ will be titrated below $50 \%$, while ensuring $\mathrm{SpO}_{2}$ greater than $90 \%$. When the subjects are suitable for SBT, they will proceed to undergo an SBT for 2 hours. During the SBT, the patients will breathe through the ventilator circuit by using flow triggering $(2 \mathrm{~L} / \mathrm{min})$ with automatic tube compensation of $100 \%$ and $5 \mathrm{cmH}_{2} \mathrm{O}$ PEEP. ${ }^{20}$ The $\mathrm{FiO}_{2}$ will be set to the same value as used before SBT. During the 2-hour periods, we will terminate the trial immediately and turn back to the ventilation mode used before the trial if the SBT fails. Patients who pass the 2-hour SBT will be extubated and followed up for the presence of postextubation respiratory distress for 48 hours. Right diaphragm excursion and bilateral diaphragm thickening fraction (DTF) will be evaluated by ultrasonography during spontaneous breathing (SB) immediately prior to SBT, and at 5, 30, 60, 90 and 120 min after the initiation of SBT. RSBI, which is calculated as the ratio of respiratory frequency to tidal volume, will be simultaneously recorded at the bedside by a respiratory nurse. The values of respiratory frequency and tidal volume can be obtained from the ventilator screen. All measurements of RSBI during $1 \mathrm{~min}$ will be obtained, and the mean value will be used for analysis. Laboratory measurements including arterial blood gas, probrain natriuretic peptide (pro$\mathrm{BNP}$ ), plasma protein, haemoglobin, chemistry profile, blood count and C-reactive protein will be obtained and recorded prior to the SBT and at the end of the SBT. If the patients present signs and symptoms such as agitation, anxiety, depressed mental status, diaphoresis, cyanosis, increased accessory muscle activity, facial signs of distress and dyspnoea during SBT, his or her arterial blood gas will also be obtained and recorded immediately. Cardiac function will be assessed by pro-BNP and echocardiography findings such as left ventricular ejection fraction (LVEF), E-point to septal separation (EPSS) and $\mathrm{e}^{\prime} / \mathrm{a}^{\prime}$ at the end of the SBT. Attending physicians are blinded to ultrasound measurements. According to the weaning outcome, subjects will be divided into two groups, the success and the failure groups.

Criteria for passing SBT are as follows: (1) the patients are cooperative; (2) $\mathrm{SpO}_{2}$ greater than $90 \%$, respiratory rate $\leq 35$ breaths per minute, heart rate $\leq 140$ breaths per minute; and (3) there is no discomfort, diaphoresis or signs of increased breathing work. Criteria for a failure of SBT are as follows: agitation and anxiety, depressed mental status, diaphoresis, cyanosis, increased accessory muscle activity, facial signs of distress, dyspnoea, $\mathrm{PaO}_{2} \leq 50-60 \mathrm{~mm}$ $\mathrm{Hg}$ on $\mathrm{FiO}_{2} \geq 0.5$ or $\mathrm{SpO}_{2}<90 \%$, arterial $\mathrm{PaO}_{2}$ of carbon dioxide $\left(\mathrm{PaCO}_{2}\right)>50 \mathrm{~mm} \mathrm{Hg}$ or an increase in $\mathrm{PaCO}_{2}$ $>8 \mathrm{~mm} \mathrm{Hg}, \mathrm{pH}<7.32$ or a decrease in $\mathrm{pH} \geq 0.07 \mathrm{pH}$ units, respiratory rate $>35$ breaths per min or increased by $\geq 50 \%$, 
heart rate $>140$ breaths per minute or increased by $\geq 20 \%$, systolic blood pressure $>180 \mathrm{~mm} \mathrm{Hg}$ or increased by $\geq 20 \%$, systolic blood pressure $<90 \mathrm{~mm} \mathrm{Hg}$ and cardiac arrhythmias. ${ }^{121}$ A successful weaning is defined as $\mathrm{SB}$ for $>48$ hours following extubation without any level of ventilator support, including non-invasive ventilation (NIV) used as a prophylactic measure after extubation. A failed weaning is defined as either SBT failure or the inability to maintain SB for at least 48 hours after extubation, including the situation in which NIV is used for patients who have been extubated but developed acute respiratory failure within 48 hours. A patient fails the weaning if he or she passes the SBT but requires endotracheal tube to protect the airway. The time from the beginning to the end of mechanical ventilation is defined as the total ventilation time. Weaning time is the time spent in partial support mode.

\section{Diaphragm ultrasound}

Three observers will be trained prior to the study. They will measure the diaphragm excursion and thickness by ultrasound as previously described. ${ }^{1422}$ Briefly, all patients will be measured in semirecumbent position with the head of bed elevated between $20^{\circ}$ and $40^{\circ}$. The right diaphragmatic excursion is measured with a $3.5-5 \mathrm{MHz}$ ultrasound probe (Mindray M9, China). The probe is placed immediately below the right costal margin along the mid-clavicular line and is directed medially, cephalad and dorsally, so that the ultrasound beam reaches perpendicularly the posterior third of the diaphragm. The liver is used as an acoustic window. First, B-mode is used to get the best image and to select the exploration line. Then M-mode is used to display the motion of the diaphragm along the selected line. During inspiration, the normal diaphragm contracts and moves caudally towards the probe; during expiration, the normal diaphragm moves cranially away from the probe; this is recorded as an upward motion of the M-mode tracing (figure 1). The diaphragm excursion is measured on the vertical axis of the tracing from the baseline to the point of maximum height of inspiration on the image. Three measurements will be recorded and averaged. All measurements will be performed during SB.

The diaphragm thickness is measured by a $10 \mathrm{MHz}$ linear ultrasound probe which is placed in the 9 th or 10th intercostal spaces and between the anterior axillary and the midaxillary lines, perpendicular to the chest wall. ${ }^{23}$ The ultrasound image is switched to the B-mode. In this area, the diaphragm is imaged as three-layer structure, including two parallel echoic lines (the diaphragmatic pleura and the peritoneal membrane) and a hypoechoic structure between them (the muscle itself). ${ }^{23}{ }^{24}$ We will freeze the image at end expiration and end inspiration. On the frozen images, the distance from the middle of the pleural line to the middle of the peritoneal line is the diaphragm thickness (figure 2). We will measure the diaphragm thickness three times on the same scan and the values will be averaged. DTF will be estimated by the following equation:

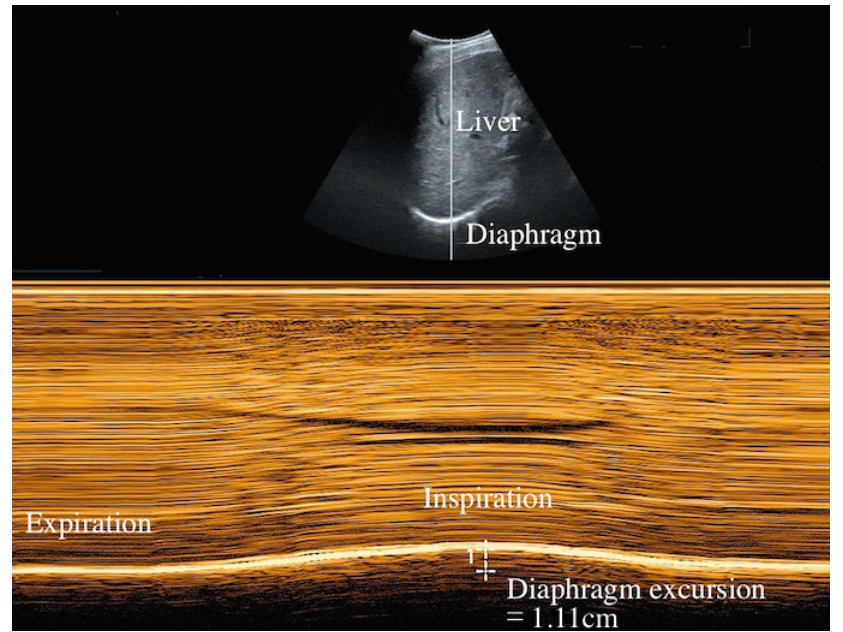

Figure 1 The liver is used as a window for the right diaphragm. During inspiration, the normal diaphragm contracts and moves caudally towards the probe; during expiration, the normal diaphragm moves cranially away from the probe; this is recorded as an upward motion of the $\mathrm{M}$-mode tracing. The diaphragm excursion is measured on the vertical axis of the tracing from the baseline to the point of maximum height of inspiration on the graph. Three measurements will be recorded and averaged.

DTF $=($ Thickness at end inspiration - Thickness at end expiration)/ Thickness at end expiration.

The DTF for each patient will be calculated as the mean of the values measured in three breaths.

All examinations will be recorded on a personal computer for subsequent blinded analysis.

\section{Follow-up study}

Diaphragm dysfunction is common in patients who are critically ill. It can be caused by multiple factors including prolonged mechanical ventilation. We first examine the serial changes in diaphragm function during an SBT with ultrasound in order to assess its value for predicting weaning outcome. Then we will measure the right diaphragm excursion and bilateral DTF during SB with bedside ultrasound on a daily basis over the first 7 days following extubation. This follow-up study will be performed in order to find whether the damage of diaphragm is reversible.

\section{Data collection}

Demographics such as sex, age on admission, BMI, underlying diseases, reasons for tracheal intubation, APACHE II and duration of mechanical ventilation will be recorded. Laboratory measurements including arterial blood gas, pro-BNP, plasma protein, haemoglobin, chemistry profile, blood count and C-reactive protein will be obtained and recorded prior to the SBT and at the end of the SBT. If the patients present signs and symptoms such as agitation, anxiety, depressed mental status, diaphoresis, cyanosis, increased accessory muscle activity, facial signs of distress and dyspnoea during SBT, his or her arterial blood gas will also be obtained and recorded immediately. Cardiac 


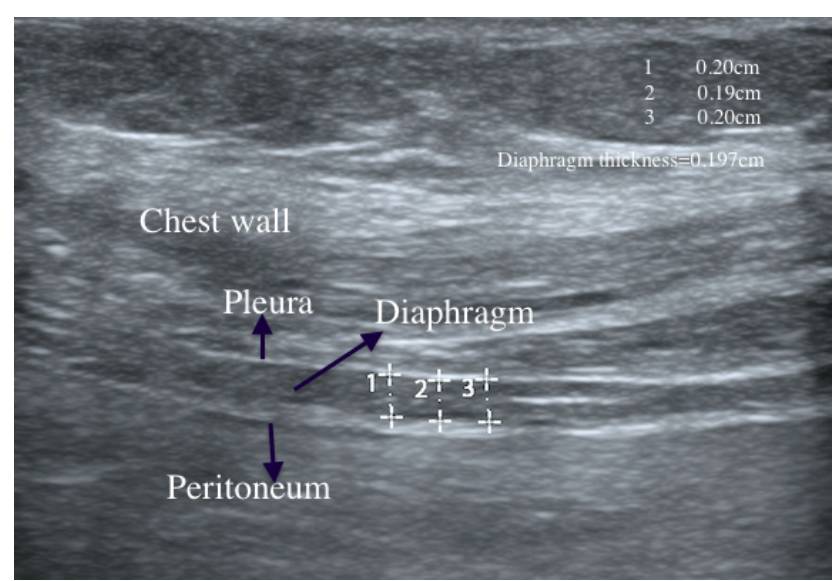

Figure 2 The diaphragm is imaged as three-layer structure, including two parallel echoic lines (the pleura and the peritoneum) and a hypoechoic structure between them (the muscle itself). The distance from the middle of the pleural line to the middle of the peritoneal line is the diaphragm thickness. We will measure the diaphragm thickness three times on the same scan and the values will be averaged. DTF will be estimated by the following equation: DTF = (Thickness at end inspiration - Thickness at end expiration) / Thickness at end expiration. The DTF for each patient will be calculated as the mean of the values measured in three breaths. DTF, diaphragm thickening fraction.

function will be assessed and recorded by pro-BNP and echocardiography findings such as LVEF, EPSS and $\mathrm{e}^{\prime} / \mathrm{a}^{\prime}$ at the end of the SBT. The RSBI, diaphragm excursion and DTF will be recorded at the 5th, 30th, 60th, 90th and 120th min of SBT. Changes in diaphragm function will be assessed by diaphragm excursion fraction (DEF) and DTF fraction (DTFF), which is calculated as the per cent change of diaphragm excursion and DTF (relative to baseline) during the SBT. DEF at time t can be calculated by the following equation:

$$
\mathrm{DEF}_{\mathrm{t}}=\left(\mathrm{DE}_{\mathrm{t}}-\mathrm{DE}_{5}\right) / \mathrm{DE}_{5} \text {, }
$$

where $\mathrm{t}$ equals $30,60,90$ and $120 \mathrm{~min}, \mathrm{DE}_{5}$ is the diaphragm excursion at $5 \mathrm{~min}$ after the initiation of SBT.

DTFF at time $\mathrm{t}$ can be calculated by the following equation:

$$
\mathrm{DTFF}_{\mathrm{t}}=\left(\mathrm{DTF}_{\mathrm{t}}-\mathrm{DTF}_{5}\right) / \mathrm{DTF}_{5} \text {, }
$$

where t equals $30,60,90$ and $120 \mathrm{~min}, \mathrm{DTF}_{5}$ is the DTF at 5 min after the initiation of SBT.

The per cent change of RSBI (relative to baseline) at time $\mathrm{t}$ can be calculated by the following equation:

$$
\mathrm{RSBIF}_{\mathrm{t}}=\left(\mathrm{RSBI}_{\mathrm{t}}-\mathrm{RSBI}_{5}\right) / \mathrm{RSBI}_{5}
$$

where t equals $30,60,90$ and $120 \mathrm{~min}, \mathrm{RSBI}_{5}$ is the RSBI at 5 min after the initiation of SBT.

Maximum per cent change in diaphragm excursion, DTF and RSBI during SBT will be recorded. The outcome is extubation failure or successful extubation.

\section{Statistical analysis}

The study aims to investigate the diagnostic performance of diaphragmatic parameters in predicting extubation failure. Thus, the estimated sensitivity and prevalence are needed for the calculation of sample size. Furthermore, we define the type I error to be 0.05 . The following equation is used to calculate the sample size:

$$
\text { Sample size }=\frac{Z_{1-\alpha / 2}^{2} \times S_{N} \times\left(1-S_{N}\right)}{L^{2} \times \text { Prevalence }},
$$

where $\alpha$ is the type I error which is assumed to be 0.05 , $\mathrm{Z}_{1-\alpha / 2}$ is $1.96, \mathrm{~L}$ is likelihood ratio which is assumed to be $0.1, \mathrm{~S}_{\mathrm{N}}$ stands for sensitivity and is assumed to be $83 \%,{ }^{18}$ and Prevalence stands for extubation failure rate and is assumed to be $33.1 \% .{ }^{25}$ A total of 164 patients are required for the study. Data will be presented as the mean and $\mathrm{SE}$, or median and IQR for continuous variables as appropriate. ${ }^{26}$ Continuous variables will be compared between the weaning success and failure groups by independent Student's t-test. Differences between the parameters with each group will be assessed by the paired Student's t-test. The $\mathrm{X}^{2}$ tests will be used to compare the differences for categorical variables. Multivariate logistic regression will be used to identify variables that can independently predict the weaning outcome, ${ }^{27}$ adjusting for variables of cardiac functions such as diastolic function and systolic function. Sensitivity, specificity, positive predictive value and negative predictive value will be calculated for diaphragm excursion, DTF, RSBI, maximum per cent change in diaphragm excursion and RSBI in predicting weaning outcome. Receiver operating characteristic (ROC) curves will be used to assess the diagnostic performance of diaphragm excursion, DTF, RSBI, maximum per cent change in diaphragm excursion and RSBI in predicting weaning outcome. Differences of the area under ROC curves will be compared using non-parametric method. Serial measured values will be analysed using serial measurement analysis of variance. To assess interobserver variability, 20 patients will be measured by two different operators. Pearson correlation analysis and Bland-Altman plotting will be performed to evaluate the reproducibility of diaphragm ultrasound studies. A two-tailed $\mathrm{p}$ value $<0.05$ is considered statistically significant. All statistical analyses will be performed using $\mathrm{R}$ software.

\section{DISCUSSION}

There are approximately $20 \%$ of patients who have difficulties in weaning from mechanical ventilation. ${ }^{1}$ Weaning outcome can be affected by a variety of factors such as cardiac dysfunction, muscle weakness, pulmonary insufficiency and electrolyte disturbances. ${ }^{28}$ As a result, single parameter is inadequate to make an accurate prediction. RSBI has been demonstrated to be accurate in predicting weaning outcome, and is now widely used in clinical practice. This index reflects the contribution of all inspiratory muscles, rather than the function of the 
diaphragm. Therefore, diaphragm fatigue can be masked by compensatory action of the other inspiratory muscle during SBT. However, the compensatory effect of non-diaphragm inspiratory muscles cannot be maintained for a long time..$^{29}{ }^{30}$ Thus, a substantial proportion of patients who pass RSBI test may be subject to weaning failure.

It is common that a patient presents a normal respiratory pattern at the beginning of an SBT, but the respiration deteriorates a few hours later. Chatila et al reported that RSBI measured at $30 \mathrm{~min}$ after initiation of SBT was superior to RSBI measured at the start of the trial as a weaning predictor. ${ }^{31}$ Another study demonstrated that RSBI measured at the end of SBT had better diagnostic accuracy. ${ }^{32}$ Also there was a study found that RSBI measured serially was more reliable to predict weaning outcomes. ${ }^{7}$ Segal et al showed that the per cent change of RSBI during SBT was better in predicting the weaning outcome. ${ }^{33}$ They concluded that the evolution of respiratory pattern, as assessed by change in RSBI, was a marker of the dynamic changes that occur during weaning that may improve the ability to predict extubation outcome. ${ }^{33}$ However, to the best of our knowledge, there is no study investigating the serial changes in diaphragm function during the SBT. We hypothesise that the changes of diaphragm function prior to RSBI are a better predictor of weaning outcome. The aim of this study is to explore whether changes in diaphragm function during SBT are a good predictor of weaning outcome. Furthermore, we attempt to investigate the timing of diaphragm function measurement which has the best diagnostic performance.

Currently the gold standard for evaluating the diaphragm function is transdiaphragmatic pressure. Other methods such as fluoroscopy and MRI have also been reported. ${ }^{345}$ However, these methods are invasive, costly and uncomfortable to patients. Also, these methods involve transportation of patients to radiology department and radiation exposure. The transportation imposes considerable risk on patients who are critically ill. Bedside ultrasound is an alternative to evaluate the diaphragm function, which is simple, rapid and non-invasive, and has been increasingly used in critical care setting. A number of studies have demonstrated that diaphragm excursion and thickening fraction could reflect the function of the diaphragm and predict the weaning outcome. ${ }^{18} 1936$ Thus, our study will employ diaphragm excursion and thickening fraction measured by ultrasound to evaluate the function of diaphragm. We will also evaluate the changes in diaphragm function by maximum per cent change in diaphragm excursion and thickening fraction during the SBT.

Contributors All the listed authors have participated actively in the study, and have seen and approved the submitted manuscript. PZ, YH and PX were responsible for the design of this study. PZ, JG, YP, YZ, XQ and SL were responsible for the drafting of the article. ZZ, $\mathrm{HC}, \mathrm{HZ}$ and JD were responsible for the revision of the article.

Funding This study received the financial support from the Department of Health of Zhejiang Province.

Competing interests None declared.
Ethics approval The ethics committee of Sir Run Run Shaw Hospital, an affiliate of Zhejiang University Medical College.

Provenance and peer review Not commissioned; externally peer reviewed.

Open Access This is an Open Access article distributed in accordance with the Creative Commons Attribution Non Commercial (CC BY-NC 4.0) license, which permits others to distribute, remix, adapt, build upon this work non-commercially, and license their derivative works on different terms, provided the original work is properly cited and the use is non-commercial. See: http://creativecommons.org/ licenses/by-nc/4.0/

(C) Article author(s) (or their employer(s) unless otherwise stated in the text of the article) 2017. All rights reserved. No commercial use is permitted unless otherwise expressly granted.

\section{REFERENCES}

1. Boles JM, Bion J, Connors A, et al. Weaning from mechanical ventilation. Eur Respir J 2007;29:1033-56.

2. Esteban A, Alía I, Ibañez J, et al. Modes of mechanical ventilation and weaning. A national survey of Spanish hospitals. The Spanish Lung Failure Collaborative Group. Chest 1994;106:1188-93.

3. El-Khatib MF, Bou-Khalil P. Clinical review: liberation from mechanical ventilation. Crit Care 2008;12:221.

4. Yang KL, Tobin MJ. A prospective study of indexes predicting the outcome of trials of weaning from mechanical ventilation. $N$ Engl $J$ Med 1991;324:1445-50.

5. Epstein SK. Etiology of extubation failure and the predictive value of the rapid shallow breathing index. Am J Respir Crit Care Med 1995;152:545-9.

6. Lee KH, Hui KP, Chan TB, et al. Rapid shallow breathing (frequencytidal volume ratio) did not predict extubation outcome. Chest 1994;105:540-3.

7. Krieger BP, Isber J, Breitenbucher A, et al. Serial measurements of the rapid-shallow-breathing index as a predictor of weaning outcome in elderly medical patients. Chest 1997;112:1029-34.

8. Johannigman JA, Davis K, Campbell RS, et al. Use of the rapid/ shallow breathing index as an Indicator of patient work of breathing during pressure support ventilation. Surgery 1997;122:737-41.

9. Barrera R, Melendez J, Ahdoot M, et al. Flow triggering added to pressure support ventilation improves comfort and reduces work of breathing in mechanically ventilated patients. $J$ Crit Care 1999;14:172-6.

10. Epstein SK, Ciubotaru RL. Influence of gender and endotracheal tube size on preextubation breathing pattern. Am J Respir Crit Care Med 1996;154:1647-52.

11. Boutou AK, Abatzidou F, Tryfon S, et al. Diagnostic accuracy of the rapid shallow breathing index to predict a successful spontaneous breathing trial outcome in mechanically ventilated patients with chronic obstructive pulmonary disease. Heart Lung 2011;40:105.10.

12. dos Reis HF, Almeida ML, da Silva MF, et al. Association between the rapid shallow breathing index and extubation success in patients with traumatic brain injury. Rev Bras Ter Intensiva 2013;25:212.7.

13. Karthika M, Al Enezi FA, Pillai LV, et al. Rapid shallow breathing index. Ann Thorac Med 2016;11:167-76.

14. Mayo P, Volpicelli G, Lerolle N, et al. Ultrasonography evaluation during the weaning process: the heart, the diaphragm, the pleura and the lung. Intensive Care Med 2016;42:1107-17.

15. Houston JG, Morris AD, Howie CA, et al. Technical report: quantitative assessment of diaphragmatic movement--a reproducible method using ultrasound. Clin Radiol 1992;46:405-7.

16. Gerscovich EO, Cronan M, McGahan JP, et al. Ultrasonographic evaluation of diaphragmatic motion. J Ultrasound Med 2001;20:597-604.

17. Jiang JR, Tsai TH, Jerng JS, et al. Ultrasonographic evaluation of liver/spleen movements and extubation outcome. Chest 2004;126:179-85.

18. Kim WY, Suh HJ, Hong SB, et al. Diaphragm dysfunction assessed by ultrasonography: influence on weaning from mechanical ventilation. Crit Care Med 2011;39:2627-30.

19. DiNino E, Gartman EJ, Sethi JM, et al. Diaphragm ultrasound as a predictor of successful extubation from mechanical ventilation. Thorax 2014;69:431-5.

20. Guttmann J, Haberthür C, Mols G, et al. Automatic tube compensation (ATC). Minerva Anestesiol 2002;68:369-77.

21. Perren A, Brochard L. Managing the apparent and hidden difficulties of weaning from mechanical ventilation. Intensive Care Med 2013;39:1885-95. 
22. Goligher EC, Laghi F, Detsky ME, et al. Measuring diaphragm thickness with ultrasound in mechanically ventilated patients: feasibility, reproducibility and validity. Intensive Care Med 2015;41:642-9.

23. Cohn D, Benditt JO, Eveloff S, et al. Diaphragm thickening during inspiration. J Appl Physiol 1997;83:291-6.

24. Ueki J, De Bruin PF, Pride NB. In vivo assessment of diaphragm contraction by ultrasound in normal subjects. Thorax 1995;50:1157-61.

25. Béduneau G, Pham T, Schortgen F, et al. Epidemiology of Weaning Outcome according to a New Definition. the WIND Study. Am J Respir Crit Care Med 2017;195:772-783.

26. Zhang Z. Univariate description and bivariate statistical inference: the first step delving into data. Ann Transl Med 2016;4:91.

27. Zhang Z. Model building strategy for logistic regression: purposeful selection. Ann Trans/ Med 2016;4:111.

28. Burns SM, Fisher C, Earven Tribble SS, et al. Multifactor clinical score and outcome of mechanical ventilation weaning trials: Burns Wean Assessment Program. Am J Crit Care 2010;19:431-9.

29. Hershenson MB, Kikuchi Y, Tzelepis GE, et al. Preferential fatigue of the rib cage muscles during inspiratory resistive loaded ventilation. $J$ Appl Physiol 1989;66:750-4.
30. Heshenson MB, Kikuchi Y, Loring SH et al. Relative strengths of the chest wall muscles. J Appl Physiol 1988:852-62.

31. Chatila W, Jacob B, Guaglionone D, et al. The unassisted respiratory rate-tidal volume ratio accurately predicts weaning outcome. Am J Med 1996;101:61-7.

32. Kuo PH, Wu HD, Lu BY, et al. Predictive value of rapid shallow breathing index measured at initiation and termination of a 2-hour spontaneous breathing trial for weaning outcome in ICU patients. $J$ Formos Med Assoc 2006;105:390-8.

33. Segal LN, Oei E, Oppenheimer BW, et al. Evolution of pattern of breathing during a spontaneous breathing trial predicts successful extubation. Intensive Care Med 2010;36:487-95.

34. Nason LK, Walker CM, McNeeley MF, et al. Imaging of the diaphragm: anatomy and function. Radiographics 2012;32:E51-E70.

35. Korin HW, Ehman RL, Riederer SJ, et al. Respiratory kinematics of the upper abdominal organs: a quantitative study. Magn Reson Med 1992;23:172-8.

36. Ferrari G, De Filippi G, Elia F, et al. Diaphragm ultrasound as a new index of discontinuation from mechanical ventilation. Crit Ultrasound J 2014;6:8. 